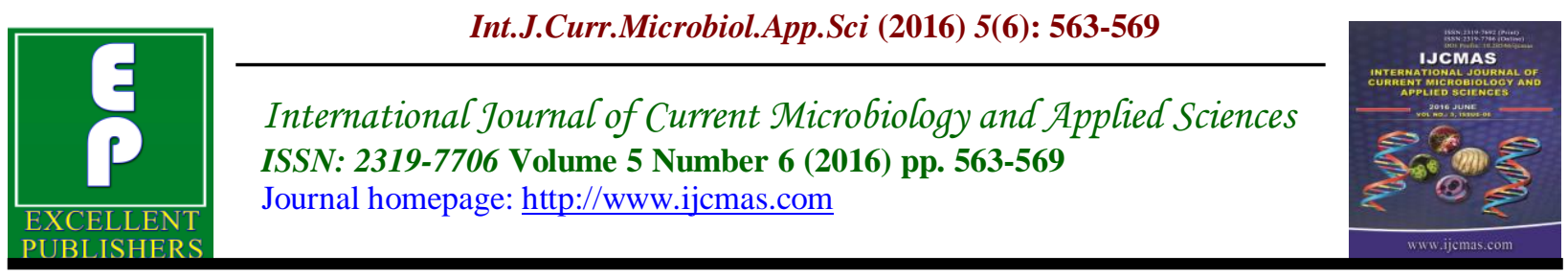

Original Research Article

http://dx.doi.org/10.20546/ijcmas.2016.506.060

\title{
Laboratory Diagnosis of Mycobacterium tuberculosis in Resource Constraint Setting: Direct Sputum Smear Microscopy is Comparable with Automated Mycobacterium Growth Indicator Tube (BACTEC MGIT 960)
}

\author{
Ochei, Kingsley Chinedum', Obeagu, Emmanuel Ifeanyi ${ }^{2}$, Odo Michael ${ }^{1}$ \\ and Ugobo Emmanuel Eteng ${ }^{3}$ \\ ${ }^{1}$ Family Health International (FHI 360) Country Office, Garki Abuja Nigeria \\ ${ }^{2}$ Diagnostic Laboratory Unit, Health Services Department, Michael Okpara University of \\ Agriculture, Umudike, Abia State, Nigeria \\ ${ }^{3}$ Initiative for People's Good Health Ugep, Cross River State, Nigeria \\ *Corresponding author
}

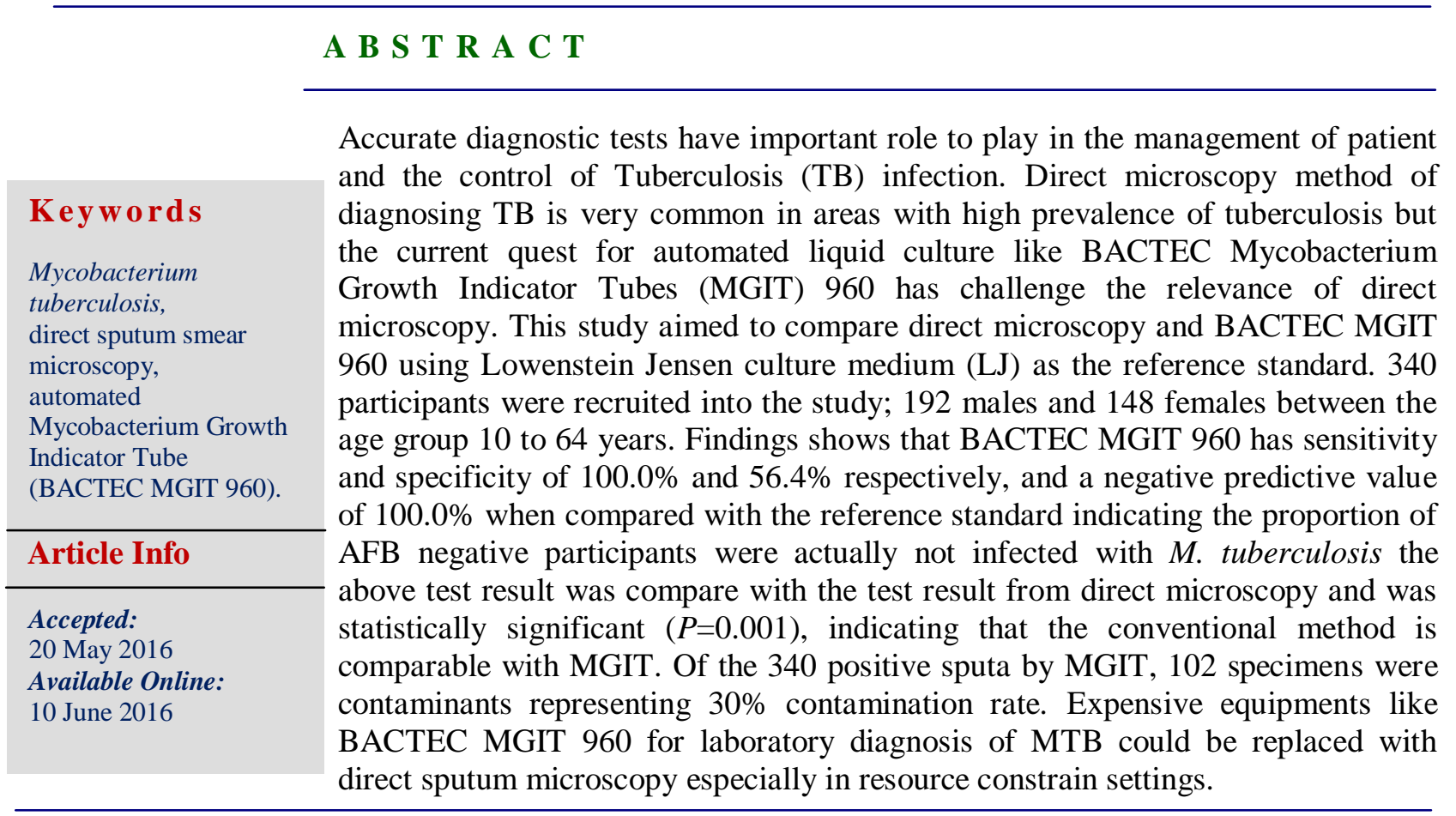

\section{Introduction}

Pulmonary Mycobacterium tuberculosis (MTB) continues to be one of the infectious diseases affecting low and medium income countries (LMIC), especially in sub-Saharan African countries. In order to successfully control the spread of MTB, cases must be detected and treated immediately and effectively. The detection of cases has always been direct sputum microscopy in LMIC, where nearly 95 percent of TB cases and 98 percent of deaths occur as a result of MTB (International standards for Tuberculosis care). Direct microscopy is a 
simple, rapid and inexpensive method which is very specific in areas with high prevalence of tuberculosis (International standards for Tuberculosis care). This method also identifies the most infectious patients and it is widely applicable in various populations with different socioeconomic levels (Steingart et al., 2006). However, the current quest for automated liquid culture like BACTEC Mycobacterium Growth Indicator Tubes (MGIT) 960 has challenge the relevance of direct microscopy.

In brief, the BACTEC MGIT 960 is an automated equipment that consists of liquid broth medium in a growth indicator tube and a device that detects the growth of the mycobacteria. The liquid medium is known to yield better recovery and faster growth of mycobacteria. This medium is made up of modified Middlebrook 7H9 broth base and reconstituted with a growth supplement (Oleic acid, Albumin, Dextrose and Catalase) and PANTA (Polymyxin B, Amphotericin B, Nalidixic acid, Trimethoprim, and Azolocillin). Also, present in the tube is an oxygen-quenched fluorochrome, tris 4, 7-diphenyl-1, 10phenonthroline ruthenium chloride pentahydrate, embedded in a silicone. During bacterial growth within the tube, the free oxygen is utilized and is replaced with carbon dioxide. As the free oxygen depletes, the fluorochrome fluorescence within the tube. The intensity of fluorescence is directly proportional to the extent of oxygen depletion and this is dependent on the growth of bacteria as well as mycobacteria (Siddiqi and Rusch-Gerdes, 2006). This system has been described as an essential for strengthening tuberculosis diagnoses, care and treatment in many countries (Siddiqi, SH and S. Rusch-Gerdes, 2006). It has been reported that, a well funded clinical laboratories can effectively detect MTB cases within 7 to 14 days using automated liquid culture system like BACTEC MGIT 960 (Luz et al., 2000).

However, there are challenges associated with the introduction of this system into laboratories in a setting like ours, as described in a systematic review (Linda et $a l .$, 2011). The major challenge has been attributed to lack of funding of clinical laboratories. In LMIC, MTB laboratories lack the required funding and human resource to support case detection using the sophisticated and expensive equipments, like the BACTEC MGIT 960. (Linda et al., 2011 and Luz et al., 2000).

There have been varied reports of the sensitivity of direct microscopy in different settings, as documented in a review (Steingart et al., 2006) and this has led to several researches into alternative methods and validation in the laboratory diagnosis of MTB (Ameh et al., 2013, Walid et al., 2011, Catharina et al., 2010, Ongkhammy et al., 2009; Maryline et al., 2008; Wah et al., 2001; Farnia et al., 2002; Frimpong et al., 2005; Merid et al., 2009). However, there seems to be less documentation on performance evaluation of direct microscopy when compared with the automated liquid culture system. This is also similar to comparing direct microscopy with the LJ culture; few studies reported this comparison to the best of our knowledge (Frimpong et al., 2005, Angeby et al., 2000, Naganathan et al., 1979,).

The primary aim of this study was to compare direct microscopy and BACTEC MGIT 960 using Lowenstein Jensen culture medium (LJ) as the reference standard. We report the differences in sensitivity, specificity, positive and negative predictive values between direct microscopy and BACTEC MGIT 960. 


\section{Materials and Methods}

\section{Settings and Patient Recruitment}

340 referred patients from DOTS clinics located in six different government owned health facilities spread across the Federal Capital Territory (FCT), Abuja in Nigeria. These facilities are Maitama District Hospital, Asokoro District Hospital, Wuse General Hospital, Gwagwalada Specialist Hospital, Kubwa General Hospital and Gwarimpa General Hospital. These hospitals are managed through the government agency, Hospital Management Board. The patients were referred to our facility, Zankli Medical Centre. These participants; male (192) and female (148) that were between the age of 10 and 64 years old were prospectively enrolled into the study between November 2004 and July 2005. A verbal informed consent was obtained from these participants and ethical approval granted by the ethical committee of FCT Hospital Management Board and Zankli Medical Centre. The participants referred from the six sites were assessed for suspected pulmonary tuberculosis. Participants that did not submit three specimens over two day's period and participants receiving anti-tuberculosis treatment were excluded from the study.

\section{Specimen Collection for Direct Microscopy}

All the 340 participants submitted three sputa specimens over two consecutive days. Thus, a total of 1,020 sputa were collected. The first specimen was collected at first visit to the referred site while the second was collected at patient's home based on the instructions given (early in the morning before brushing teeth) and the third specimen, when the patient brought the morning specimen. Instructions were given on how to produce an appropriate specimen for diagnosis of pulmonary TB. The two spot specimens were produced by patients in an open and free ventilated area of the facility. We performed direct smear on all the specimens collected.

\section{Direct Microscopy}

1 by $2 \mathrm{~cm}$ smears were made from the purulent part of the sputum, air-dried and heat fixed on a hot plate at $85^{\circ} \mathrm{C}$ for $2-3$ minutes and stained with $\mathrm{ZN}$ method $(1 \%$ filtered carbol-fuchsin and $0.1 \%$ malachite green or methylene blue).

\section{Microscopic Examination and Interpretation}

The smears were read using oil immersion lens (x100) of ordinary light microscope by experienced microscopist who were blinded to culture results. Positive and negative smears were defined according to the National Tuberculosis and Leprosy Control Program (NTBLCP) Acid Fast Bacilli (AFB) grading (Table 1). A patient is reported smear positive for tuberculosis if at least $1-9$ AFB is seen in 100 high power fields. For the purpose of this study, we only considered a definitive case detection and not necessarily the grading. Hence, we reported on number of patients diagnosed with active M. tuberculosis infection.

\section{Sputum Decontamination, Culture and Identification of M. Tuberculosis complex}

Sputum for culture was randomly selected from three specimens of every participant. To $5 \mathrm{ml}$ of sputum was added an equal volume of $4 \%$ sodium hydroxide in a $30 \mathrm{ml}$ screw-cap tube. This was capped tightly and shaken to digest the sputum; thereafter allowed to stand at room temperature for 15 minutes with occasional shaking. The 
mixture was centrifuged at 3,000 X $\mathrm{g}$ for 15 minutes. The supernatant was carefully decanted and the deposit was re-suspended with $15 \mathrm{ml}$ of sterile normal saline and recentrifuged again at the same rate.

Solid media $-0.5 \mathrm{ml}$ of the sediment from the processed sputa were inoculated onto LJ agar slope and incubated at $37 \pm 2{ }^{0} \mathrm{C}$ and observed daily for the first three days for possible contamination and thereafter regularly examined at weekly interval for 6 10 weeks for the isolation of $M$. tuberculosis. Positive and negative growth controls were always included using wild strains of $M$. tuberculosis complex and sterile distilled water respectively.

Liquid media - After inoculation of each tube with $0.5 \mathrm{ml}$ of the processed sputa, the tubes were incubated at $37^{\circ} \mathrm{C}$ in the BACTEC MGIT 960 and monitored automatically every one hour for increased fluorescence. The cultures were tested until positive or for 42 days.

Identification of mycobacteria Identification of mycobacteria was based on colony morphology, colony pigmentation, rate of growth on solid media, the results of biochemical tests; nitrate reductase test, catalase heat-labile test. $M$. tuberculosis complex was only differentiated from nontuberculous mycobacteria and no further identification tests were done.

\section{Definitions}

1) Reference standard is positive and negative LJ culture.

2) Positive MGIT culture was considered as positive signal and positive AFB smear.

3) Negative MGIT culture was considered as negative signal at 42 days and negative AFB smear.
4) Contaminant is when AFB smear of a positive MGIT culture (growth at 42 days) proved to be negative.

\section{Statistical Analysis}

Sensitivity, specificity, negative and positive predictive values were calculated using standard definitions (1). Statistical analysis was performed with Stata SE software version 11 (Stata Corp LP, College station TX, USA). A p-value of $<0.05$ was considered statistically significant.

\section{Results and Discussion}

340 participants recruited during the 8 months period study profile, is shown in Figure 1. When compared with the reference standard, BACTEC MGIT 960 has a sensitivity and specificity of $100.0 \%$ and $56.4 \%$ respectively, and a negative predictive value of $100.0 \%$ (Table 2) indicating the proportion of AFB negative participants were actually not infected with M. tuberculosis when tested with BACTEC MGIT 960. Sensitivity and specificity of direct microscopy, when compared with BACTEC MGIT 960 is statistically significant $(P=0.001)$, indicating that the conventional method is comparable with MGIT. Of the 340 positive sputa by MGIT, 102 specimens were contaminants representing $30 \%$ contamination rate.

One of the advantages of comparing diagnostic method with another, especially with reference standard is to established test performance characteristics (Evaluating diagnostics, TDR). This is helpful in determining diagnostic properties of the method like sensitivity and specificity before its introduction into the laboratory. 
Table.1 Guide to AFB microscopy interpretation.

\begin{tabular}{ll}
\hline Number of AFB & Recording/reporting \\
\hline No AFB in at 100 fields & Negative \\
1-9 AFB seen in 100 fields & Actual number \\
1-9 AFB in 10 fields & + \\
1-9 AFB per field & ++ \\
$>10$ AFB per field & +++ \\
\hline
\end{tabular}

Table.2 Diagnostic tests characteristics of BACTEC MGIT 960 and direct smear determined when compared with the reference standard. PPV - positive predictive value, NPV - negative predictive value, $\mathrm{CI}$ - confidence interval

\begin{tabular}{lcccccc}
\hline \multicolumn{2}{c}{ Direct Microscopy } & \multicolumn{2}{c}{ BACTEC MGIT 960 } & Difference & \\
\hline $\begin{array}{l}\text { Diagnostic } \\
\text { accuracy }\end{array}$ & $\mathrm{n} / \mathrm{N}$ & $\%(95 \% \mathrm{CI})$ & $\mathrm{n} / \mathrm{N}$ & $\%(95 \% \mathrm{CI})$ & $\%(95 \% \mathrm{CI})$ & P value \\
\hline & & & & & $-15.1(-21.9 ;-$ & \\
Sensitivity & $90 / 106$ & $84.9(78.1 ; 91.7)$ & $106 / 106$ & $100(100 ; 100)$ & $8.3)$ & $<0.001$ \\
Specificity & $226 / 234$ & $96.6(94.3 ; 98.9)$ & $132 / 234$ & $56.4(50.1 ; 62.8)$ & $40.2(33.4 ; 46.9)$ & $<0.001$ \\
PPV & $90 / 98$ & $91.8(86.4 ; 97.3)$ & $106 / 208$ & $51.0(44.2 ; 57.8)$ & $40.9(32.2 ; 49.6)$ & $<0.001$ \\
NPV & $226 / 242$ & $93.4(90.3 ; 96.5)$ & $132 / 132$ & $100(100 ; 100)$ & $-6.6(-9.7 ;-3.5)$ & 0.0025 \\
\hline
\end{tabular}

This study therefore, compared direct microscopy with the BACTEC MGIT 960, using the solid medium as the reference standard. Our result indicates that in the absence of the automated liquid culture system, direct microscopy could be utilized, as indicated in the statistical analysis in Table 2. In another study in Zimbabwe by Aper et al.(2003), the direct microscopy was reported to be comparable with BACTEC MGIT 960, statistically. The study reported an agreement between the two diagnostic methods as $72 \%(\mathrm{p}<0.0001)$. While the sensitivity and specificity was reported as $67.5 \%$ and $95.5 \%$ respectively. This study reported a higher sensitivity when compared with the study in Zimbabwe. This observation could have been as a result of increased sample size utilized in this study.

Of concern, is the contamination rate of MGIT observed in this study and it is more than the generally accepted $2-5 \%$ for LJ culture and it is an outlier when compared with studies that utilized MGIT culture versus techniques like BACTEC 460 , LJ and Ogawa; contamination rate vary from $1.2 \%$ to $20.1 \%$. The studies concluded that contamination with rich MGIT medium is more common when compared with other culture media. This contamination rate could have contributed to the reduced specificity and it is a disadvantage of the MGIT system. However, this limitation cannot be compared with the advantage of a shorter detection time than any other culture method but it is not cost effective as already reported (Apers et al., 2003). Although, this study did not evaluate the cost effectiveness of the MGIT system but it is obvious that this diagnostic method is likely not realistic in a laboratory that lacks the human resource and infrastructure to support it. Hence, direct smear microscopy will continue to be the pillar for MTB detection in these resource constraint settings. Also, the reported variations in sensitivity rate; ranging from 
more than $80 \%$ in some settings and 20 to $60 \%$ (Steingart et al., 2006) of this method have not prevented tuberculosis control programs in LMIC countries from utilizing this approach. So, for as long as the laboratories in these settings continue to experience lack of funding, direct microscopy will still remain the only method for diagnosis of pulmonary tuberculosis. It is therefore not surprising, that the current guidelines of World Health Organization and the International Union against Tuberculosis and Lung Disease specify the importance of MTB diagnosis by microscopic examination of sputum samples.

In conclusion, expensive equipments like BACTEC MGIT 960 for laboratory diagnosis of MTB could be replaced with direct sputum microscopy in a setting that is poorly funded and lack human resources.

\section{References}

Angeby, K..A, Alvarado-Galvez, C., PinedaGarcia, L.\& Hoffner, S.E.(2000). Improved sputum microscopy for a more sensitive diagnosis of pulmonary tuberculosis. Int $J$ Tuberc Lung Dis. 4: 684-87.

Apers, L., Mutsvangwa, J., Magwenzi, J.(2003). A comparison of direct microscopy, the concentration method and the mycobacteria growth indicator tube for the examination of sputum for acid-fast bacilli. Int $J$ Tuberc Lung Dis.7: 376-81.

Catharina, C.B., Pamela, N., Doris, H., Mark, P.N., Shubhada, S., Fiorella, K., Jenny, A., Rasim, T., Robert, B., Roxana, R., Ana, M., Martin, J., Sean, M.O., David, H.P., Sabine, R.G., Eduardo, G., Camilla, R., David, A.\& Perkins, M.D. (2010). Rapid molecular detection of tuberculosis and rifampin resistance. N. Engl. J. Med. 363(11):1005-1015.

Drewe, J.A., Alexandra, J. T., Neil, J. W.\& Richard, J. D. (2010). Diagnostic accuracy and optimal use of three tests for tuberculosis in Live Badgers. PLoS One. 5(6): e11196.

Farnia, P., Mohammadi, F.,, Zarifi Z., Tabatabee, D.J., Ganavi, J., Ghazisaeedi, K., Farnia, P.K., Gheydi, M., Bahadori, M., Masjedi, M.R.\& Velayati, A.A. (2002). Improving sensitivity of direct microscopy for detection of acid-fast bacilli in sputum: use of chitin in mucus digestion. J. Clin. Microbiol. 40:508511.

Frimpong, E.H., Adukpo, R.\& OwusuDarko, K. (2005). Evaluation of two novel Ziehl-Neelsen methods for tuberculosis diagnosis. West Afr. J. Med. 24:316-320.

Linda M. P., Akos, S., Cristina, G., Evan, L., Paramasivan,C.N., Alash'le, A., Steven, S., Giorgio, R. and John, N. (2011). Laboratory diagnosis of tuberculosis in resource-poor countries: challenges and opportunities. Clin. Microbiol. Rev. 24(2): 314-350.

Luz ,C., Lee, T. S., Gilman, R. H., Sheen, P., Spellman, E., Lee, E. H., Berg, D. E.\& Montenegro-James, S. (2000). Rapid, efficient detection and drug susceptibility testing of Mycobacterium tuberculosis in sputum by microscopic observation of broth cultures. J.Clin.Microbiol.38(3): 1203-1208.

Maryline, B., Andrew, R., Willie, G., Laramie, G., Francis, V.\& Philippe, J.G. (2008). Bleach sedimentation. An opportunity to optimize smear microscopy for tuberculosis diagnosis in settings of high prevalence of HIV. 
CID. 46:1710-1716.

Merid, Y., Yassin, M.A., Yamuah, L., Kumar, R., Engers, H.\& Aseffa, A. (2009). Validaiton of bleach treated smears for the diagnosis of pulmonary tuberculosis. Int. J. Tuber. Lung Dis. 13:136-141.

Naganathan, N., Ganapathy, K.T.\& Rajalakshmi, R.(1979). Evaluation of sputum smears prepared by diff erent methods. Indian J Med Res . 69: 893900.

Nature reviews. Microbiology September 2006. Evaluating diagnostics, Nature publishing.

Ongkhammy, S., Amstutz, B. H.\& Buisson, Y. (2009). The bleach method improves the detection of pulmonary tuberculosis in Laos. Int. J. Tuberc. Lung. Dis. 13(9):1124-1129

Siddiqi, S.H. and Rusch-Gerdes,S. (2006),,. MGIT procedure manual. Foundation for Innovative New Diagnostics, Geneva, Switzerland.

Steingart, K. R., Vivienne, N., Megan, H.,
Hopewell, P.C., Ramsay, A., Cunningham, J., Urbanczik, R., Perkins, M.D., Aziz, M.A., \& Pai, M. (2006). Sputum processing methods to improve the sensitivity of smear microscopy for tuberculosis: a systematic review. Lancet Infect Dis 6, 664-674.

Wah, W.A., Mar, M.N., Ti, T.\& Win, M. (2001). Improved method of direct microscopy for detection of acid-fast bacilli in sputum. Southeast Asian J. Trop. Med. Public Health 32(2):390393.

Walid, B.S., Hedi, H. \& Jalel, B. (2011). Immunochromatographic $\quad \mathrm{IgG} / \mathrm{IgM}$ test for rapid diagnosis of active tuberculosis. Clin. Vaccine Immunol. 18(12):2090-2094.

World Health Organization, Global tuberculosis $\quad \operatorname{report}(2011)$. http://www.who.int/tb/publications/gl obal_report/2011/gtbr11_full.pdf. accessed on August 15, 2013.

\section{How to cite this article:}

Ochei, Kingsley Chinedum, Obeagu, Emmanuel Ifeanyi, Odo Michael and Ugobo Emmanuel Eteng. 2016. Laboratory Diagnosis of Mycobacterium tuberculosis in Resource Constraint Setting: Direct Sputum Smear Microscopy is Comparable with Automated Mycobacterium Growth Indicator Tube (BACTEC MGIT 960). Int.J.Curr.Microbiol.App.Sci. 5(6): 563-569. doi: http://dx.doi.org/10.20546/ijcmas.2016.506.060 